clinic visitors. In view of the extensive resistance against doxycyclin and ciprofloxacin, these antibiotics are not appropriate treatment options for gonorrhoea; instead, extended spectrum cephalosporins are advised.

Disclosure of interest statement The study is funded by Indonesian government through Beasiswa Unggulan (The Excellence Scholarship Program), Ministry of Education and Culture Republic of Indonesia and Public Health Service (GGD) of Amsterdam, The Netherlands. The authors declare that there is no conflict of interest.

\section{P05.06 PROLONGED INFECTION OF PHARYNGEAL GONORRHOEA AFTER TREATMENT WITH CEFTRIAXONE}

1,2,3 IPY Hananta* ${ }^{2,3}$ MF Schim van der Loeff, ${ }^{3} \mathrm{AP}$ van Dam, ${ }^{1} \mathrm{H}$ Soebono, ${ }^{2,3} \mathrm{HJC}$ de Vries. ${ }^{1}$ Department of Dermatology and Venereology Faculty of Medicine Universitas Gadjah Mada, Yogyakarta, Indonesia; ${ }^{2}$ Academic Medical Center, Universiteit Van Amsterdam, The Netherlands; ${ }^{3}$ Public Health Service (GGD) of Amsterdam, The Netherlands

\subsection{6/sextrans-2015-052270.292}

Introduction Previous studies reported that in a considerable proportion of pharyngeal gonorrhoea cases treated with extended-spectrum cephalosporins, the infection remains detectable after several weeks. We examined the occurrence of prolonged pharyngeal gonorrhoea infections after treatment with ceftriaxone at a sexually transmitted infection (STI) outpatient clinic in Amsterdam.

Methods A retrospective cohort study was conducted based on routine electronic medical records at the STI clinic of the Public Health Service (GGD) of Amsterdam. Eligible for inclusion were: adults diagnosed with pharyngeal gonorrhoea between January 2012 and July 2013, who were treated with ceftriaxone (500 mg IM), and who returned for a test of cure (TOC) between 7 and 21 days after treatment.

Definitive diagnosis of gonorrhoea was based on Gen-Probe Aptima-Combo 2 Assay $^{\mathrm{TM}}$ using Tigris DTS ${ }^{\mathrm{TM}}$ system. Some patients also received additional antibiotics with ceftriaxone.

Information on patients' characteristics and clinical history were available, but data on sexual re-exposure after treatment were not.

Results In the study period, 880 pharyngeal gonorrhoea cases were diagnosed; 290 cases (32.9\%) returned for a TOC visit and were eligible (255 males and 35 females, median age 34 and 25 years, respectively). In 17 cases (5.9\%) N. gonorrhoeae infection was detected again. Prolonged infection was not associated with gender $(\mathrm{p}=0.49)$ or age $(\mathrm{p}=0.87)$, but appeared to be associated with sex work (OR $=3.24[95 \%$ CI 0.83-12.45], $\mathrm{p}=0.07$ ). Prolonged infection was significantly more common among those who were treated with ceftriaxone only vs a combined-regimen $(\mathrm{OR}=4.07,[95 \% \mathrm{CI}$ 0.90-18.39]; $\mathrm{p}=0.048$ ).

Conclusion Prolonged pharyngeal gonorrhoea infection after appropriate treatment was not uncommon, and was more often observed in those who were treated with ceftriaxone only. This could be the result of re-infection after treatment or of treatment failure possibly due to poor tissue penetration. Treatment failure due to antimicrobial resistance seems unlikely.

Disclosure of interest The study is fully funded by Public Health Service (GGD) of Amsterdam, The Netherlands. The authors declare that there is no conflict of interest.

\section{P05.07 NEISSERIA GONORRHOEAE MULTIANTIGEN SEQUENCE TYPING (NG-MAST) OF ISOLATES COLLECTED FROM STD PATIENTS IN DELHI, INDIA}

${ }^{1} S$ Sood, ${ }^{1} \mathrm{~N}$ Mahajann, ${ }^{1} \mathrm{R}$ Singh, ${ }^{1} \mathrm{~A}$ Kapil, ${ }^{1} \mathrm{BK}$ Das, ${ }^{2} \mathrm{~V}$ Sreenivas, ${ }^{3} \mathrm{HK}$ Kar, ${ }^{4} \mathrm{VK}$ Sharma. ${ }^{1}$ Department of Microbiology, All India Institute of Medical Sciences, New Delhi; ${ }^{2}$ Department of Biostatistics, All India Institute of Medical Sciences, New Delhi; ${ }^{3}$ Department of Dermatology, STDs and Leprosy, P. G. I. M. E. R \& Dr Ram Manohar Lohia Hospital, New Delhi; ${ }^{4}$ Department of Dermatology \& Venereology, All India Institute of Medical Sciences, New Delhi

\subsection{6/sextrans-2015-052270.293}

Background Neisseria gonorrhoeae multiantigen sequence typing (NG-MAST) is a highly discriminatory technique for assessing the genetic diversity of $N$. gonorrhoeae and has also been put forward as a tool for predicting specific antimicrobial resistance (AMR) phenotypes. Therefore, the present study was undertaken to investigate the molecular epidemiology of N. gonorrhoeae isolates using NG-MAST in Delhi and to examine if it can be used as a means for predicting AMR. This is the first such research performed in this country.

Methods 100 consecutive gonococcal isolates between April 2010 to October 2013 were investigated. Antimicrobial susceptibility testing was done using disc diffusion method and E test and the results interpreted using the breakpoint criteria of CDS technique. NG-MAST was performed as described previously. WHO N. gonorrhoeae reference strains F, G, K-P were used as controls. Association between NG-MAST sequence type (ST) and antimicrobial susceptibility was probed using chi-square and fisher's exact tests.

Results Rates of resistance to classical antibiotics were high. Decreased susceptibility to ceftriaxone (MIC $0.032-0.25 \mu \mathrm{g} / \mathrm{ml}$ ) was demonstrated in 5\% while azithromycin resistance (MIC $\geq 1$ $\mu \mathrm{g} / \mathrm{ml}$ ) was seen in $4 \%$ isolates. N. gonorrhoeae isolates were assigned into 60 different STs and $43(71.6 \%)$ have not been reported previously to the international database. The most common ST was 6058 (21\%), followed by ST 9774 (4\%), ST9875 (4\%), ST9783 (4\%) and ST2990 (3\%). The majority of the STs $(76.6 \%)$ were represented by a single isolate. There was significant association between ST6058 and resistance to penicillin (p $=0.00)$ and tetracycline $(\mathrm{p}=0.002)$. In all the other antibiotics, no association was found.

Conclusion Our work reflects a highly diversified gonococcal population in Delhi. Further, NG-MAST has a limited applicability as a tool for predicting AMR in our region. A detailed investigation on a large number of representative isolates may provide insight into sexual networks in the city.

Disclosure of interest statement None.

\section{P05.08 BETA-LACTAM ANTIBIOTICS INDUCE PROTEIN EXPRESSION CHANGES IN NEISSERIA GONORRHOEAE REVEALED BY A PROTEOMIC APPROACH}

'S Nabu*, ${ }^{1} \mathrm{C}$ Isarankura-Na-Ayudhya, ${ }^{1,2} \mathrm{R}$ Lawung, ${ }^{3} \mathrm{P}$ Isarankura-Na-Ayudhya, 'V Prachayasittikul. 'Department of Clinical Microbiology and Applied Technology, Faculty of Medical Technology, Mahidol University, Bangkok, Thailand; ${ }^{2}$ Center of Medical Laboratory Service, Faculty of Medical Technology, Mahidol University, Bangkok, Thailand; ${ }^{3}$ Department of Medical Technology, Faculty of Allied Health Science, Thammasat University, Pathumthani, Thailand

10.1136/sextrans-2015-052270.294 